\title{
Active Gas Replenishment and Sensing of the Wetting State in a Submerged Superhydrophobic Surface
}

Ben P. Lloyd*t, Philip N. Bartlett ${ }^{\ddagger}$, Robert J. K. Wood ${ }^{\dagger}$

${ }^{\dagger}$ National Centre for Advanced Tribology at Southampton (nCATS), University of Southampton, SO17 1BJ, UK

${ }^{\ddagger}$ Chemistry, University of Southampton, SO17 1BJ, UK

Keywords: Dewetting, wetting transition, low drag, Green surfaces

\begin{abstract}
Previously superhydrophobic surfaces have demonstrated effective drag reduction by trapping a lubricious gas layer on the surface with micron-sized hydrophobic features. However, prolonged reduction of drag is hindered by the dissolution of the gas into the surrounding water. This paper demonstrates a novel combination of superhydrophobic surface design and electrochemical control methods which allow quick determination of the wetted area and a gas replenishment mechanism to maintain the desirable gas filled state. Electrochemical impedance spectroscopy is used to measure the capacitance of the surface which is shown to be proportional to the solid/liquid interface area. To maintain a full gas coverage for prolonged periods the surface is held at an electrical potential which leads to hydrogen evolution. In the desired gas filled state the water does not touch the metallic area of the surface, however after gas has dissolved the water touches the metal which closes the electrochemical circuit causing hydrogen to be produced replenishing the gas in the surface and returning to the gas filled state; in this way the system is self-actuating. This type of surface and electrochemical control shows promise for applications where the gas filled state of superhydrophobic surfaces must be maintained when submerged for long periods of time.
\end{abstract}

\section{Introduction}

Superhydrophobic surfaces have shown great promise reducing skin friction drag in laminar 1,2 and turbulent flows ${ }^{3,4}$ by supporting a layer of gas on the solid surface. ${ }^{5}$ However recently experiments have shown the dissolution of the gas film into the surrounding water means the low drag properties are short lived..$^{6-8}$ Without a full coverage of gas on the surface in the Cassie state, low drag cannot be realised. 
Methods to quantify the gas volume and to replenish gas as it dissolves are required to progress the use of superhydrophobic surfaces from the laboratory into more applied areas.

When drops of water are deposited onto superhydrophobic surfaces in the majority of cases the gas beneath the drop is still connected to the atmosphere so dissolution does not lead to a change in the wetting state. However some surfaces trap gas beneath drops where the wetting properties could be altered by the dissolution of the gas. ${ }^{9}$ When fully submerged, all superhydrophobic surfaces are susceptible to gas dissolution induced wetting transitions. Some experiments looking at the drag reducing properties of gas laden surfaces have neglected to carefully measure the wetting state, indeed, adding means to gain in situ information on the gas film is certainly a challenging problem in larger scale towing tank tests. The unknown wetting state of surfaces in such tests is a probable cause of the discrepancy between published results in this area. If such surfaces are to be introduced into real applications the automated monitoring of the wetting state will be vital to their operation.

The sensing of the wetting state of superhydrophobic surfaces in laboratory conditions is often achieved using optical microscopy, the presence of gas is observed as silver coloured reflection in the light from the microscope. $8,10,11$ This method is simple and quick, however it only gives qualitative rather than quantitative information of the presence of a gas layer. In some cases the raw image is processed to give a binary black and white image to which wetted and non wetted areas are assigned. This is likely to overly simplify the problem as it does not consider the transition between the two states.

Surfaces with relatively large features (on a scale of hundreds of micrometres) and regular patterns, such as posts and gratings, lend themselves to easy optical determination of the wetting state. However when the features are smaller, approaching the wavelength of light, or irregular it becomes more difficult to use optical methods to identify a wetted surface from one with a full gas coverage. ${ }^{12}$ However with sophisticated microscopes resolutions down to $\sim 200 \mathrm{~nm}$ are possible. Specialised systems such as laser scanning confocal microscopy and the use of fluorescent dye labelled water can show the position of the three phase contact line exactly within the surface structures but are much slower and more difficult to carry out in situ. ${ }^{13}$

At a superhydrophobic surface the solid area exposed to the liquid increases as the gas is displaced; electrochemical methods are well suited to measuring this change in wetted area. A considerable benefit of electrochemical area determination is that it does not rely on direct observation of the surface through the liquid, as is the case with optical methods, and it can be used remotely or with turbid solutions. However the method is restricted to conducting surfaces. Two groups have shown this type of measurement to be effective. Tuberquia et al. measured the impedance of their superhydrophobic rough polymethylene surface as it was immersed in water. ${ }^{12}$ Ethanol was added to the water in a stepwise fashion which reduced the liquid surface tension progressively wetting the surface. The impedance was measured throughout the process and decreased as more of the surface was wetted. Dhindsa et al. measured the capacitance of a superhydrophobic surface as the gas film was displaced by electrowetting, as the surface was wetted the capacitance increased. ${ }^{14}$ 
Even when the water is saturated with dissolved air the gas held within a superhydrophobic surface with a geometry capable of significant drag reduction in large scale fluidic systems will start to dissolve when submersed more than a few centimetres. ${ }^{7}$ This means that if superhydrophobic surfaces are to be successful at reducing drag for prolonged periods a method by which gas is actively replaced is a necessity. Kim and Lee designed and made a surface to reverse the wetting transition caused by dissolution of the gas or water pressure. ${ }^{15}$ Hydrogen gas was generated electrochemically by the splitting of the water when it contacted regions of the surface. They showed that for the spread of the gas film between pillars, or along a grating, the bottom of the surface must itself be superhydrophobic - which they achieved using a nanostructured texture covered in a layer of Teflon. However once this layer became wetted reversal of the wetting transition was not possible.

Several methods have been developed to reverse the wetting transition of drops on a superhydrophobic surface. These include locally reduced pressure from above the surface ${ }^{16}$, vibration, ${ }^{17}$ droplet coalescence, ${ }^{18}$ back pressure from beneath the surface, ${ }^{19}$ and high temperature ${ }^{20,21}$ However these have not been shown to be appropriate for reversing the wetting transition when the superhydrophobic surface is fully submersed.

In this paper we use the templated electrodeposition method developed by Bartlett et al., ${ }^{22}$ which has been shown previously to encourage the entrapment of gas $^{9}$ and has the advantage of not requiring standard lithographic methods, to fabricate a nickel surface that has an hexagonal close packed array of $3 \mu \mathrm{m}$ diameter hemispherical cavities. The outer surface of this structure is then coated with a thin polymer layer that is electrically insulating and hydrophobic. This design produces a surface that is selfactuating and which allows the easy and continuous measurement of the wetted area of the surface when it is used as the working electrode in a three electrode system. The novel surface design means that in the desired Cassie state the water rests on the polymer film on top of the cavities and does not touch the metal walls inside of the cavities; in this state the electrochemical circuit is open so no current flows. However if the water starts to penetrate into the cavities, starting the transition to the undesirable wetted state, the electrochemical circuit is closed and hydrogen is generated by electrolysis of water inside the cavities replenishing the gas and returning the surface to the Cassie state. This self-actuation, afforded by the novel surface geometry, allows both quantification of the wetting state, by simultaneous electrochemical impedance measurements, and replenishment of the gas. Unlike methods that rely on heating the replenishment of gas requires relatively low power to maintain the Cassie state.

\section{Experimental Section}

To fabricate the superhydrophobic surface templated electrodeposition was employed, ${ }^{22}$ the process is shown in figure 1 . The template consists of a close packed array of $3 \mu \mathrm{m}$ diameter polystyrene spheres (Polysciences) formed by an evaporation driven self assembly process. A microcell was made using a parafilm (Pechiney Plastic) spacer between a glass coverslip and a gold coated microscope slide into which a solution containing the spheres was injected. The assembly was placed into an oven at $40{ }^{\circ} \mathrm{C}$ and the solution left to evaporate with the sample held horizontally. This drives the self assembly process of the spheres into a close packed monolayer. The microcell was removed from the gold coated slide and nickel was then electrodeposited around the template to a height of $1.5 \mu \mathrm{m}$ (half the sphere diameter). 
Electrodeposition of nickel was carried out potentiostatically at $-0.9 \mathrm{~V}$ against a saturated calomel reference electrode from a Watts bath (nickel (II) chloride hexa-hydrate $240 \mathrm{~g} \mathrm{~L}^{-1}$, nickel (II) sulphate hexahydrate $45 \mathrm{~g} \mathrm{~L}^{-1}$, boric acid $30 \mathrm{~g} \mathrm{~L}^{-1}$, saccharin $\left.2 \mathrm{~g} \mathrm{~L}^{-1}\right)$. The charge passed throughout the electrodeposition was carefully monitored so the deposition could be stopped once the desired film height had been achieved. A film of poly(dimethylsiloxane) (PDMS) was spin coated on the nickel deposit and template; 10\% PDMS (Dow Corning Sylgard 184) in tert-butanol at 8000 RPM for 5 min using a spin coater (WS-650 by Laurell Technologies) gave a film thickness of $\sim 80 \mathrm{~nm}$. The spheres were dissolved using tetrahydrofuran. The resulting surface had a projected area of $0.1 \mathrm{~cm}^{2}$ and was composed of exposed metal cavities topped with a thin PDMS layer, figure 2. Images of the surface were obtained using a scanning electron microscope (Philips XL 30 ESEM).

A three electrode system was used, controlled by a potentiostat (Autolab PGSTAT302N). The potential was measured against a saturated calomel reference electrode. The sample was used as the working electrode and a large area platinum wire mesh was used for the counter electrode. They were arranged facing one another $1 \mathrm{~cm}$ apart. An aqueous 0.1 molar phosphate buffered solution at $\mathrm{pH} 7$ and $20 \mathrm{C}^{\circ}$ was used for the electrolyte. A schematic of the electrochemical experiments is shown in the Supplementary Information section.

The dissolved gas concentration was controlled by vacuum degassing when required and was measured using a dissolved oxygen probe (DS300 by Eutech). Parafilm was used to cover the solution surface once the desired dissolved gas concentration had been achieved to reduce gas exchange to and from the atmosphere.
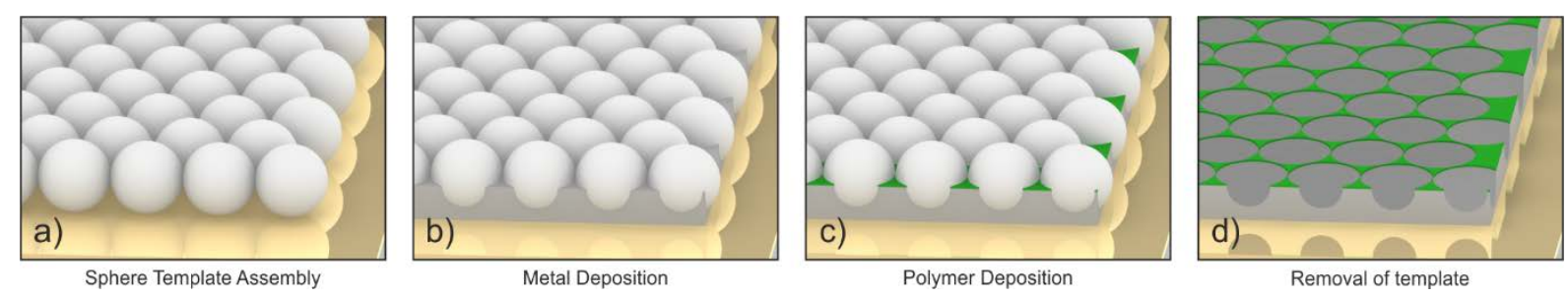

Figure 1 Schematic showing the steps involved to fabricate the desired surface. a) Assembly of $3 \mu \mathrm{m}$ polystyrene latex spheres in to a close packed monolayer. b) Electrodeposition of nickel to half the sphere height. c) Spin-coating deposition of poly(dimethysiloxane). d) Removal of polystyrene spheres by dissolving in tetrahydrofuran. 

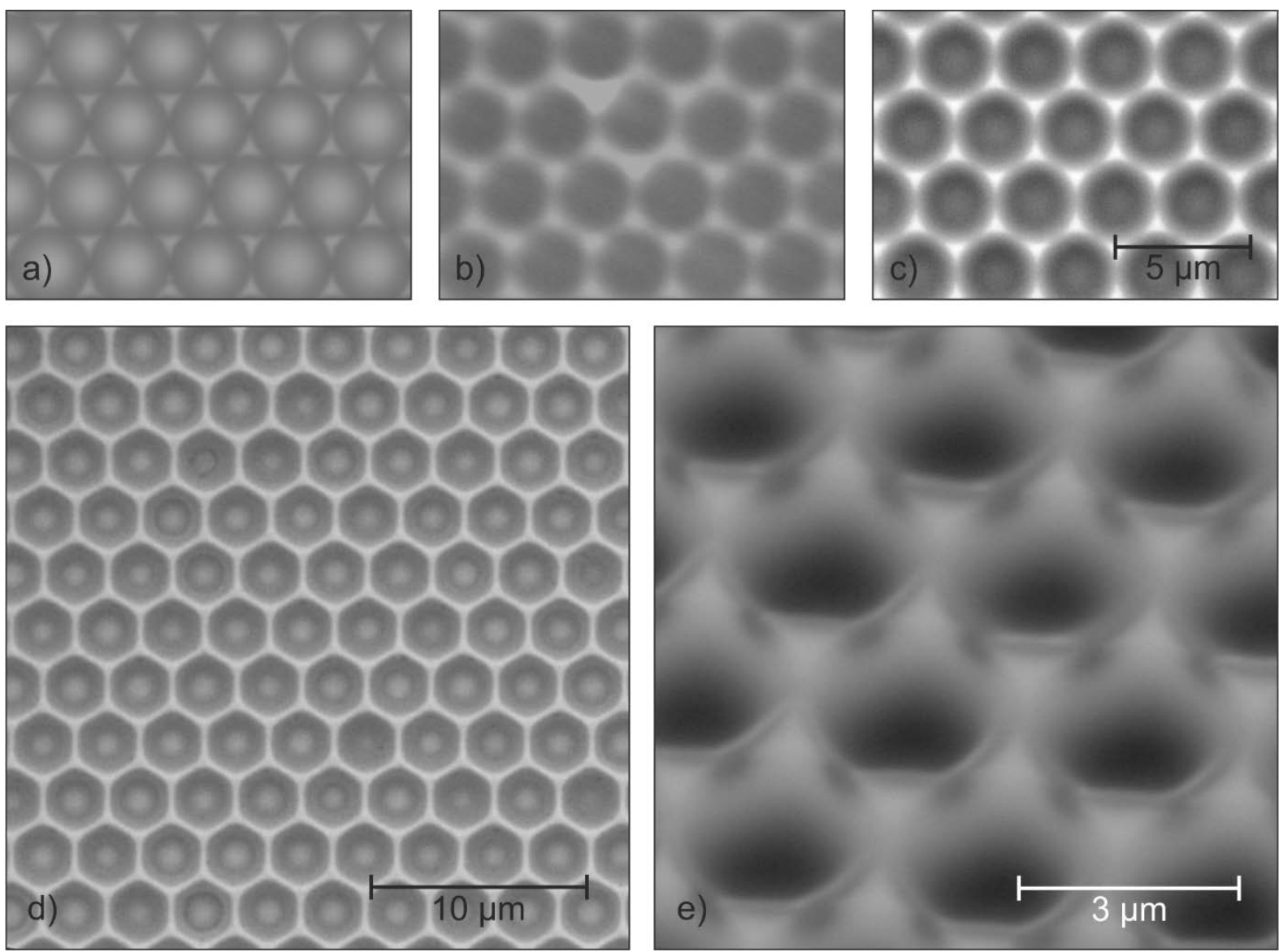

Figure 2 Scanning electron microscope images of the surface. a) Polystyrene spheres with nickel electrodeposited to half the sphere diameter. b) PDMS spin coated over polystyrene sphere template and nickel. c) PDMS and nickel after the sphere template has been dissolved away. d) The surface showing long range order in finished surface. The PDMS is seen as the lighter coloured area between cavities. e) The surface shown at higher magnification and at an angle.

\section{Results \& Discussion}

The surface was fabricated using a modified templated electrodeposition method (figure 1). ${ }^{22}$ The superhydrophobic surface consists of hexagonally close packed metallic cavities with a hydrophobic and electrically insulating polymer layer covering the top of the surface between the cavities. The template was formed using $3 \mu \mathrm{m}$ diameter polystyrene spheres around which nickel was electrodeposited to half the sphere height. A thin film ( $80 \mathrm{~nm}$ ) of poly(dimethylsiloxane) was then spin coated onto the surface. The film selectively covers the top of the surface. The spheres were then removed by dissolution in tetrahydrofuran. The resulting structure is regular with a smooth surface and uniform thickness (figure 2). The advancing and receding contact angles of water drops on the surface were $150^{\circ} \pm 5^{\circ}$ and $120^{\circ} \pm 15^{\circ}$ respectively, agreeing with our previous work considering the wetting properties on similar surfaces. ${ }^{9}$

The superhydrophobic surface was submerged to a depth of $1 \mathrm{~cm}$ in $0.1 \mathrm{M} \mathrm{pH} 7$ phosphate buffered electrolyte and used as the working electrode in a three electrode system. Using this system two 
functionalities were explored. Firstly the measurement of the wetted area of the cavities as the trapped gas within the cavities dissolves into the surrounding liquid and secondly the replenishment of the gas to maintain the Cassie state as the gas dissolves.

There are many ways to measure the area of an electrode by electrochemical means. ${ }^{23}$ However several methods require the introduction of a redox probe into the solution, or oxidise and reduce the metal surface, which would lead to material loss. Measurement of the capacitance using electrochemical impedance spectroscopy (EIS) avoids both of these issues and is also fast to measure $(<30 \mathrm{~s})$.

Impedance measurements were taken at 15 frequencies between 1000 and $10000 \mathrm{~Hz}$ using a potential modulation of $\pm 5 \mathrm{mV}$ (rms) about a potential of $-0.1 \mathrm{~V}$ vs. SCE. No significant faradic processes occur at this potential.

A Nyquist plot of a flat nickel electrode is shown in figure 3 a), the circuit is assumed to be a resistor and capacitor in series (RC), where the resistance is dominated by the resistance of the solution and the capacitance by the electrical double layer capacitance. The plot shows a deviation from the response of an ideal RC circuit, which would give a vertical line. Such deviation is typical of electrochemical systems and can be treated as a constant phase element ${ }^{24}$ where the impedance, $Z$, is given as

$$
Z=R+\frac{1}{(j \omega)^{\alpha} C}
$$

where $R$ is the resistance, $j$ is the imaginary number $\sqrt{-1}, \omega$ is the frequency in rad $\mathrm{s}^{-1}, \alpha$ is an exponent describing the deviation from ideal capacitive behaviour and $C$ is the capacitance. The measured data was fitted to equation 1 to obtain the resistance and capacitance. The value of $\alpha$ was found to be 0.99 for this system. This approach was used to obtain all of the following capacitance measurements.
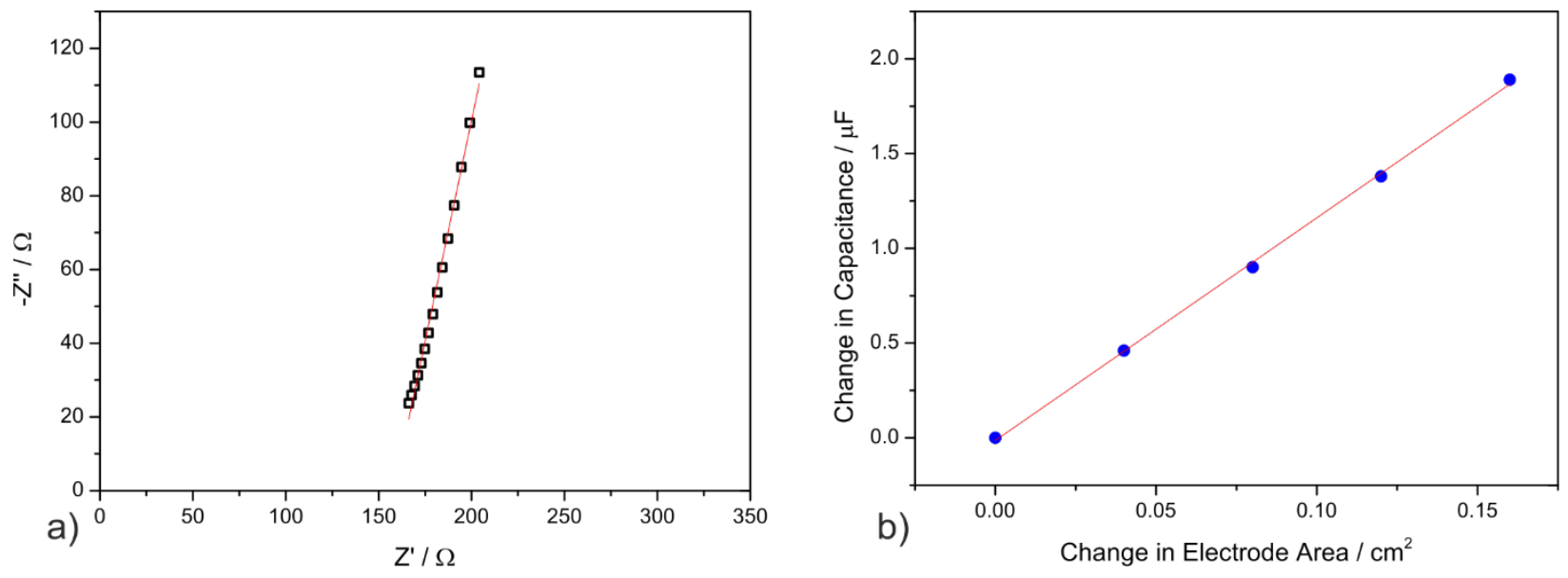

Figure 3 a) Nyquist plot for a nickel electrode in $0.1 \mathrm{M}$ phosphate buffer at - $0.1 \mathrm{~V}$ vs SCE reference b) Change in capacitance plotted against change in electrode area. The blue circles show the capacitance measurements and the red line is a linear fit of the data. 
A micro adjuster was used to submerse a $4 \mathrm{~mm}$ wide rectangular nickel electrode in $1 \mathrm{~mm}$ intervals to increase its wetted area and capacitance measurements were taken after each step. The relationship between electrode area and capacitance is displayed in Figure $3 \mathrm{~b}$ ). The results show that they are directly proportional to one another. The red line shows a linear fit (R-squared of $>0.99$ ) with a gradient of 11.8 $\mu \mathrm{F} \mathrm{cm}^{-2}$ which agrees well with values in the literature for the double layer capacitance. ${ }^{23}$ This demonstrates that measurement of the capacitance using EIS under these conditions is suitable for the reliable determination of electrode area.

Previous work by Tuberquia et al. ${ }^{12}$ and Dhindsa et al. ${ }^{14}$ detected changes in electrochemical response between the Cassie and fully wetted states. The transition between the states was promoted by reduction of the liquid surface energy by addition of methanol and electrowetting respectively. In this work the gas is allowed to dissolve into the surrounding solution. This allows us to demonstrate a functional method to measure wetted area and to determine the lifetime of the trapped gas at the surface with respect to dissolution.

The cavity surface was submersed in the solution and the wetted area was measured as the gas within the cavities dissolved, exposing a greater solid/liquid interface. Capacitance measurements were started immediately after submersion. The term specific surface capacitance is used for this measured value, it is the capacitance per projected unit area. The change in specific surface capacitance and wetted fraction against time is shown for two conditions; where the solution had be pre-treated to $80 \%$ air saturation and $100 \%$ air saturation. As discussed determination of the wetting state through optical methods is not always possible and in this case the visible difference in wetting states was imperceptible.

The $80 \%$ saturation condition is shown in figure 4 a). As expected initially the specific surface capacitance is low as the cavities are gas filled, but it increases quickly as the gas dissolves readily into the undersaturated solution, wetting the cavities. A stable specific surface capacitance reading is reached when the surface is completely wetted. This shows that the fully wetted sample has a specific surface capacitance of $20 \mu \mathrm{F} \mathrm{cm}^{-2}$. The same value was obtained after full degasing of the surface under vacuum which confirms this is indeed the fully wetted state. The wetted fraction of the sample is calculated by dividing a given specific surface capacitance by this fully wetted specific surface capacitance of $20 \mu \mathrm{F} \mathrm{cm}{ }^{-2}$, so the wetted fraction is 1 for the fully wetted state and 0 for the Cassie state. The wetted fraction is shown in the right $y$-axis for both conditions in figure 4 . Note that the fully wetted specific surface capacitance of $20 \mu \mathrm{F} \mathrm{cm}^{-2}$ agrees well with the calculated geometric surface area of the structured surface ( $\sim 1.8$ times the projected area) multiplied by the value of $11.8 \mu \mathrm{F} \mathrm{cm}^{-2}$ for a flat surface.

Under $100 \%$ air saturated conditions, figure $4 \mathrm{~b}$ ), we see again the initial capacitance is near zero and then increases with time. The rate of change in capacitance is much slower than the $\sim 80 \%$ saturation condition, as expected. In this case the experiment was terminated at $\sim 400$ minutes with a wetted fraction of $\sim 0.5$. 

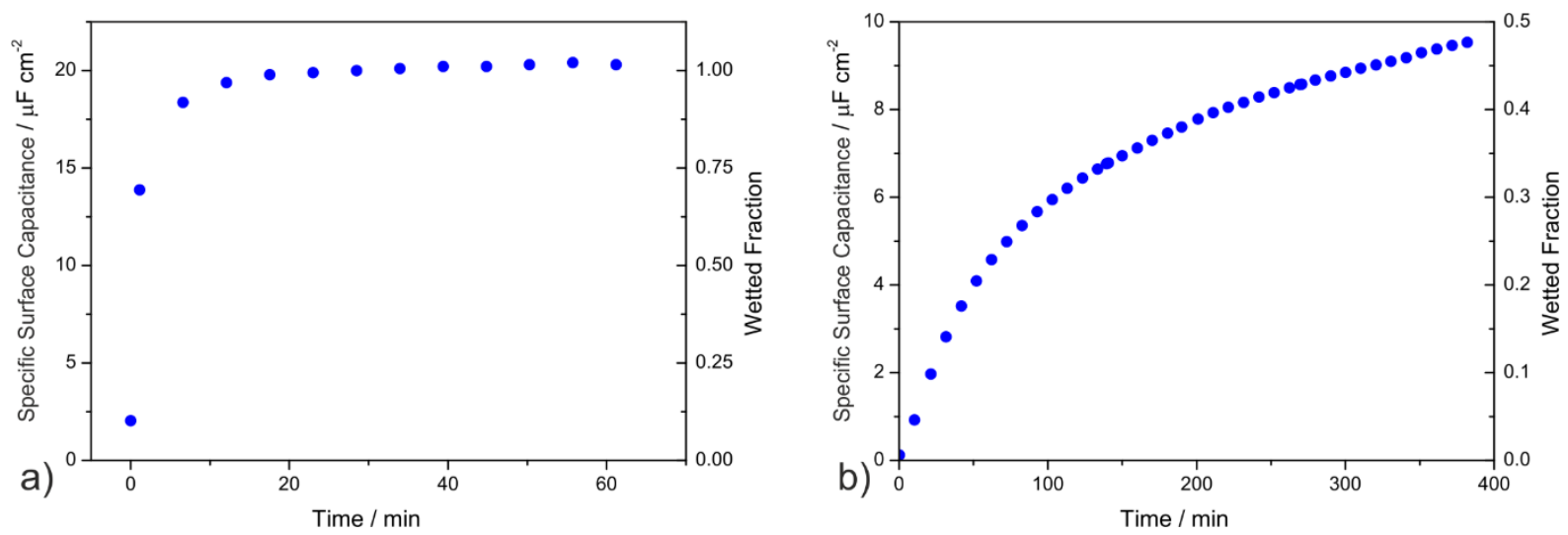

Figure 4 Graphs showing the specific surface capacitance and wetted fraction over time as the gas trapped in the cavities dissolves. In a) using a solution at $80 \%$ gas saturation and b) $\mathbf{1 0 0} \%$ gas saturation conditions in $0.1 \mathrm{M}$ phosphate buffer.

The PDMS coated areas also contribute in parallel to the double layer capacitance. However their contribution to the total capacitance is dwarfed by the nickel double layer contribution - the initial value in $100 \%$ saturated conditions where the nickel area is at a minimum is $0.1 \mu \mathrm{F} \mathrm{cm}^{-2}$ which is $0.5 \%$ of the fully wetted state where the nickel area is its largest.

These experiments demonstrate the susceptibility of the gas layer to dissolution even in $100 \%$ saturated conditions and show the need for an active method to replace gas once it is lost.

To replace gas which is lost through dissolution hydrogen was evolved directly from the surrounding water which means there is no limit to the total volume of gas the surface can produce. Hydrogen evolution occurs at the negatively charged cathode, meaning oxidation of the surface is also prevented. A cyclic voltammogram of a flat nickel electrode is shown in figure 5 , where the onset of hydrogen evolution occurs around $-0.75 \mathrm{~V} v$ s. SCE. Based on the geometry of the surface and the measured voltammetry we estimate that the regeneration of all of the trapped hydrogen at the surface requires $\sim 1 \mathrm{~mJ} \mathrm{~cm}^{-2}$, neglecting any dissolution, and would take around 2 seconds. 


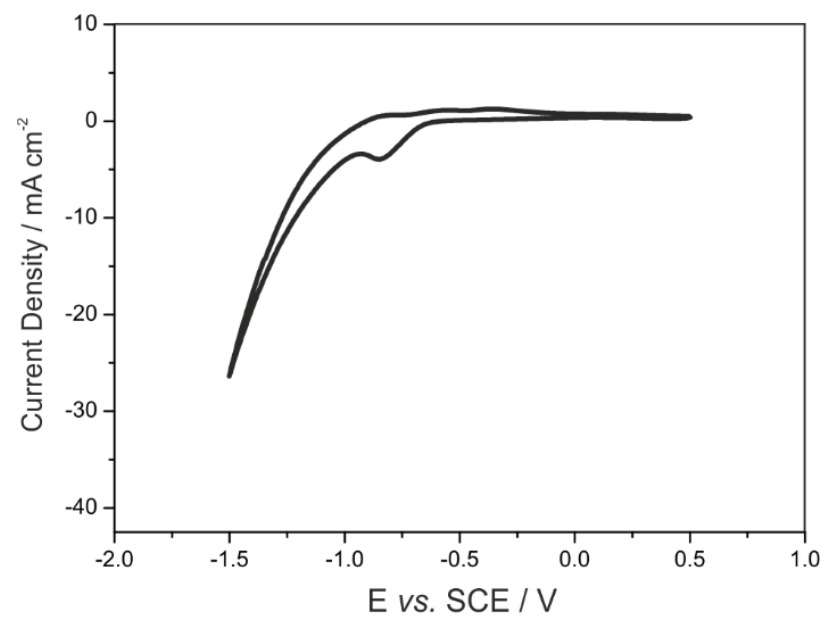

Figure 5 Cyclic voltammetry of nickel in $0.1 \mathrm{M} \mathrm{pH} 7$ phosphate solution at $50 \mathrm{mV} \mathrm{s}^{-1}$. Hydrogen evolution starts at $-0.75 \mathrm{~V}$ and rate of production increases as the potential scans more negative.

The sample was submersed into saturated water and the surface was set to $-1.5 \mathrm{~V} v$ s. SCE. Measurements of the capacitance were taken every 5 minutes to measure the wetted area of the surface. Hydrogen evolution was temporarily ceased during the capacitance measurements, figure 6 .

Throughout the experiment the current density remains fairly constant between -100 and $-200 \mu \mathrm{Acm}^{-2}$ as hydrogen is evolved when required. The specific surface capacitance also remains constant at around 40 $\mathrm{nF} \mathrm{cm}{ }^{-2}$ indicating that the cavities are kept dry by the production of hydrogen throughout the experiment. A sketch of the topping up process is shown in figure 7.

The novel geometry of the surface regulates the gas production, self actuating when needed and self limiting when not. In the desired Cassie state the water only touches the insulating PDMS layer and the electrochemical circuit is open, but in the undesirable situation where water intrudes into the metal cavity the circuit is closed. Fig 6 b) shows the current transients at higher resolution. There is an initial larger current due to charging of the double layer and a larger metal area exposed after the $30 \mathrm{~s}$ EIS measurement when hydrogen production ceased. The current density then reaches a plateau after $\sim 30$ s.

From the current density we approximate the rate of hydrogen production to be $\sim 1.9 \times 10^{-11} \mathrm{~m}^{3} \mathrm{~s}^{-1} \mathrm{~cm}^{-2}$ This assumes $100 \%$ faradaic efficiency and that all hydrogen is in the gas state, however much of the hydrogen will be dissolved in the solution. From the graph in figure $4 \mathrm{~b}$ ) showing dissolution of air from the cavities into the $100 \%$ saturated solution the initial rate of dissolution is approximated to be $\sim 1.2 \mathrm{x}$ $10^{-14} \mathrm{~m}^{3} \mathrm{~s}^{-1} \mathrm{~cm}^{-2}$. This $\sim 1000$ fold difference is likely due to the fact that the water is completely unsaturated with respect to hydrogen and therefore it dissolves at a very high rate compared to when the cavities are filled with air. The surface consumes a power of $~ 2.5 \mu \mathrm{W}$ per centimetre squared to maintain the Cassie state. This experiment demonstrates that by holding the surface at $-1.5 \mathrm{~V} v$ s. SCE a full gas coverage can be successfully maintained when it would have otherwise been lost through dissolution. 

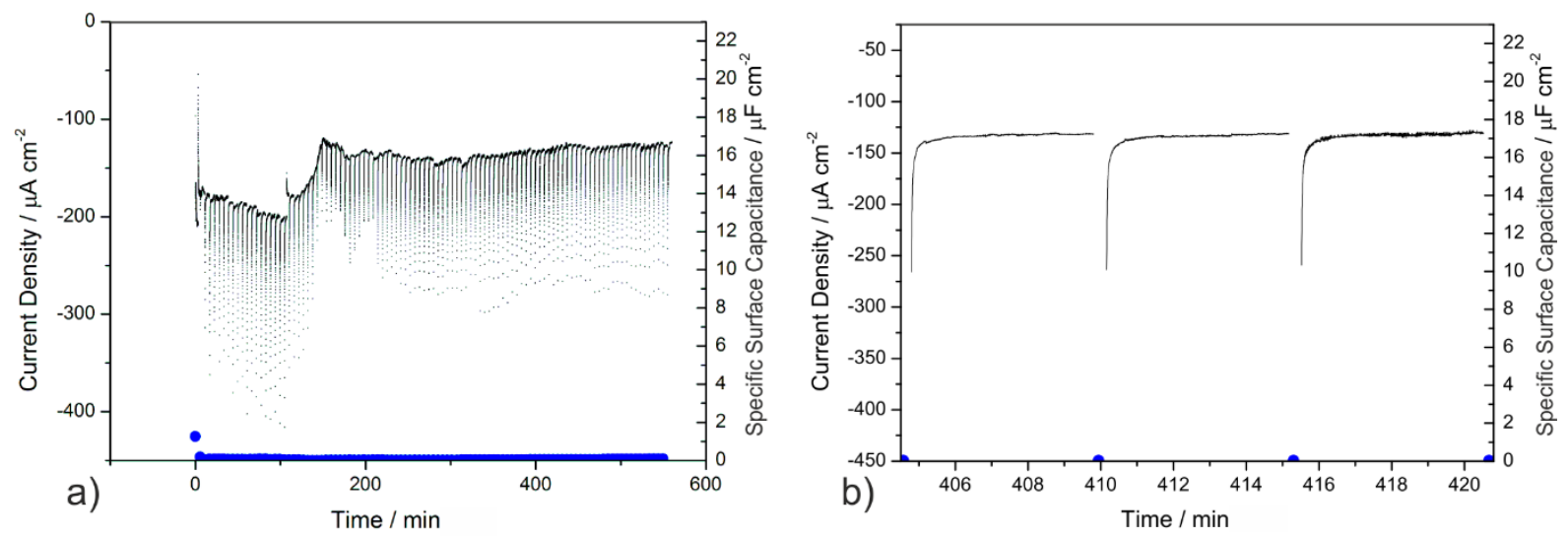

Figure 6 a) Graph displaying the current density (black line) and capacitance density (blue circles) for gas retention in the surface at $-1.5 \mathrm{~V}$ vs. SCE. b) The topping up process at higher resolution. Measurements of the specific surface capacitance were taken every 5 minutes during which time hydrogen evolution was stopped.

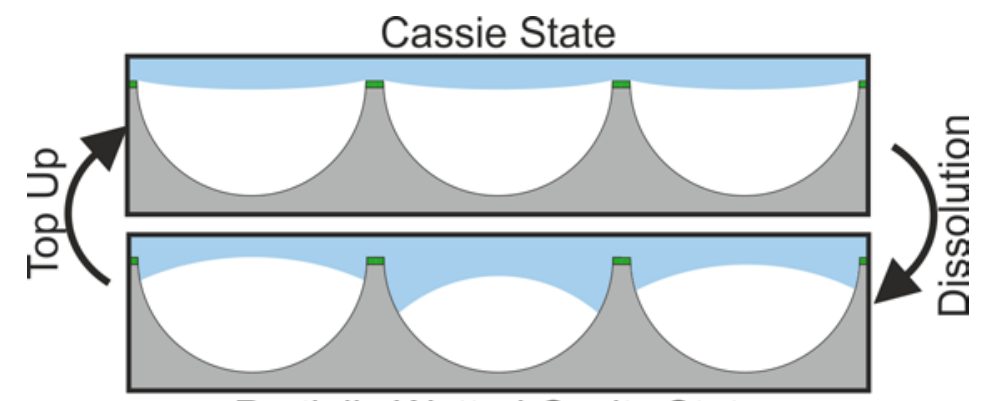

Partially Wetted Cavity State

Figure 7 The topping up process. The surface is submerged into the water and takes the Cassie wetting state. Some of the gas dissolves and the cavities become partially wetted. As the water contacts the metal inside the cavities the electrochemical circuit closes leading the evolution of hydrogen gas which tops up the cavities until the Cassie state is regained.

To attempt to recover the Cassie state from a completely wetted state the surface and bath were held under vacuum to remove all gas from the surface. The same potential of $-1.5 \mathrm{~V} v$ s. SCE was then applied with capacitance measurements taken every $30 \mathrm{~s}$.

Figure 8 a) shows the current density and specific surface capacitance from this experiment. The initial specific surface capacitance of $\sim 20 \mu \mathrm{F} \mathrm{cm}{ }^{-2}$ shows the surface starts the experiment in a completely wetted state. After the first $30 \mathrm{~s}$ of hydrogen evolution there is a small decrease in specific surface capacitance indicating that a fraction, about 0.1 , of the surface was covered by gas however after this it does not decrease further, and in fact shows a modest increase throughout the rest of the experiment. This is because the solution in the cavities, where the hydrogen is electrochemically generated, becomes supersaturated with hydrogen but the hydrogen gas bubbles nucleate and grow not on the hydrophilic metal electrode surface but on the hydrophobic layer of PDMS on the outside of the structure. This is consistent with work in the literature that shows preferential nucleation of gas bubbles on the 
hydrophobic insulation surrounding an electrode..$^{25}$ The $~ 10 \%$ decrease in the capacitance is accounted for by the blocking effect of the large gas bubbles at the surface. The current density is much larger than in the previous experiment, around 65 times greater, due to the increased metal area exposed.

In this experiment, when the potential was applied bubbles of hydrogen could be observed streaming from the surface. Microscope imaging of this process was not possible as the viewing window was quickly obscured by the cloud of bubbles. The majority of the surface remains fully wetted and the Cassie state is not generated, however a few larger bubbles (around $350 \mu \mathrm{m}$ in diameter) are found, figure $8 \mathrm{~b}$ ).
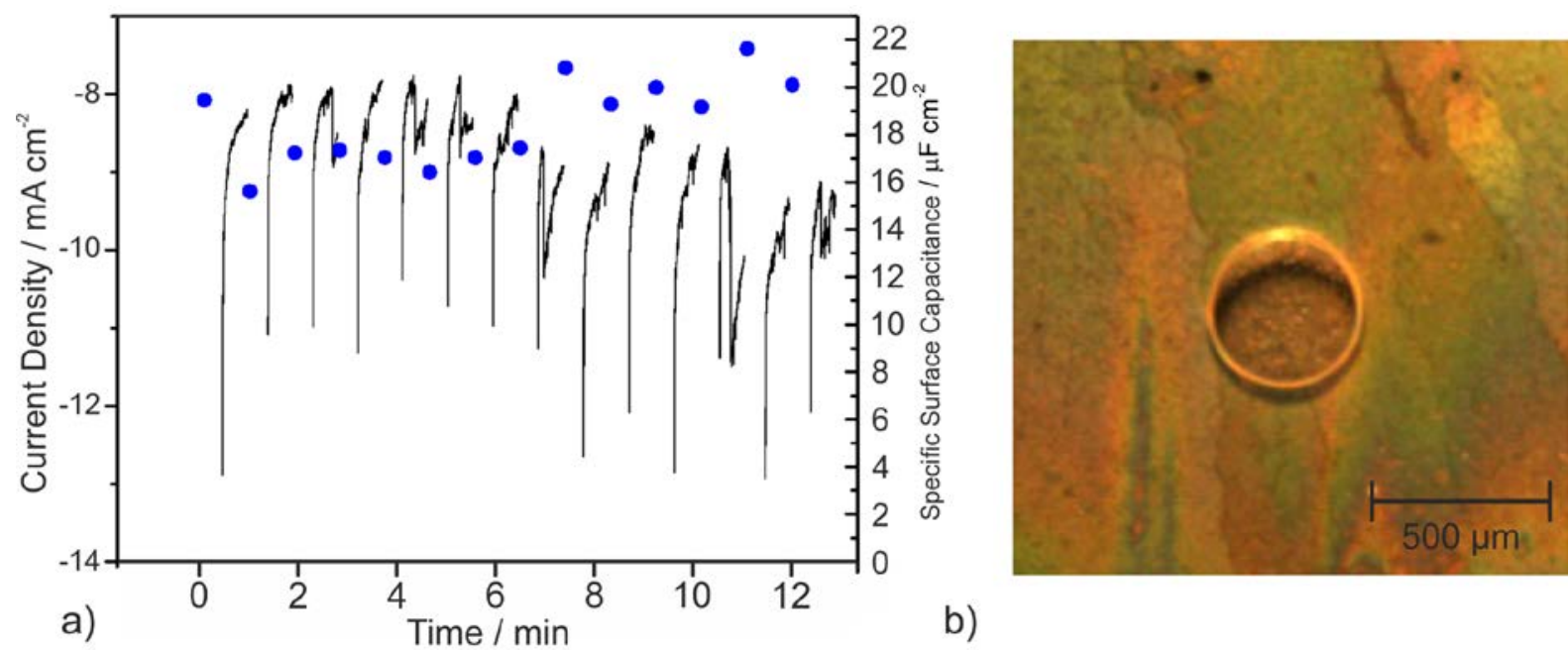

b)

Figure 8 a) Graph showing current density and specific surface capacitance when hydrogen was evolved at -1.5 V vs. SCE in $0.1 \mathrm{M}$ phosphate buffer solution after the surface had been fully wetted. b) An optical microscope image of the surface after the experiment showing the presence of large bubbles.

There seems to be a consistent theme in the response of the surface presented here and the surface of Lee and Kim. ${ }^{15}$ When a pre-existing gas domain is present on the surface, as in the wetted case of Lee and Kim where a film of gas remains within the nanostructured base of the structure, addition of more gas to this domain allows the Cassie state to be regenerated, provided the geometry is appropriate. However if the surface is totally wetted the nucleation of new gas does not occur at the correct location for full dewetting of the surface. In this case we speculate that nucleation of hydrogen bubbles occurs on the hydrophobic PDMS layer rather than inside the nickel cavities.

\section{Conclusions}

We have demonstrated a novel superhydrophobic self-actuating surface consisting of a close packed hexagonal array of hemispherical nickel cavities topped with an insulating, hydrophobic layer of PDMS. When used as an electrode in an electrochemical system the wetted fraction of the surface can be continuously measured in situ using electrochemical impedance spectroscopy. By following changes in the electrochemical impedance over time we are able to measure the rate at which the gas within the 
cavities dissolves into the surrounding water. By holding the surface at a potential at which hydrogen evolves by electrolysis of water the surface becomes self-actuating - as the gas dissolves from the cavities it is automatically replenished. This process is relatively efficient requiring $\sim 2.5 \mu \mathrm{W} \mathrm{cm}{ }^{-2}$ to maintain the gas within the cavities in our experiments.

This work could inform the design of low drag surfaces whose performance does not deteriorate due to loss of gas. The current size of the features, $3 \mu \mathrm{m}$ diameter cavities and $\sim 90 \%$ projected gas coverage, is not expected to be large enough to produce a significant reduction to drag in large scale systems but does demonstrate principles that might be applied in other systems. Assuming geometry based slip length predictions hold, ${ }^{26}$ this surface could be applied to microfluidic and other microelectromechanical devices where meaningful drag reduction could occur.

\section{Acknowledgments}

This work was funded by the Green Tribology Platform Grant from the EPSRC (EP/J001023/1). BPL acknowledges help from Dr Terry Harvey (nCATS, University of Southampton) with generating figure 1. PNB gratefully acknowledges the receipt of a Wolfson Research Merit award.

\section{References}

1. C.-H. Choi and C.-J. Kim, Phys. Rev. Lett., 2006, 96, 066001.

2. N. J. Shirtcliffe, G. McHale, M. I. Newton and Y. Zhang, ACS Appl. Mater. Interfaces., 2009, 1, 13161323.

3. R. J. Daniello, N. E. Waterhouse and J. P. Rothstein, Phys. Fluids., 2009, 21, 085103.

4. H. Park, G. Sun and C.-J. C. Kim, presented in part at the Micro Electro Mechanical Systems (MEMS), 2013 IEEE 26th International Conference on, Taipei, Jan. 2013.

5. J. P. Rothstein, Annu. Rev. Fluid. Mech., 2010, 42, 89-109.

6. P. Lv, Y. Xue, Y. Shi, H. Lin and H. Duan, Phys. Rev. Lett., 2014, 112, 196101.

7. M. Xu, G. Sun and C.-J. Kim, Phys. Rev. Lett., 2014, 113, 136103.

8. R. Govardhan, G. Srinivas, A. Asthana and M. Bobji, Phys. Fluids., 2009, 21, 052001.

9. B. P. Lloyd, P. N. Bartlett and R. J. Wood, Langmuir, 2015, 31, 9325-9330.

10. M. S. Bobji, S. V. Kumar, A. Asthana and R. N. Govardhan, Langmuir, 2009, 25, 12120-12126.

11. R. Poetes, K. Holtzmann, K. Franze and U. Steiner, Phys. Rev. Lett., 2010, 105, 166104.

12. J. C. Tuberquia, W. S. Song and G. K. Jennings, Anal. Chem., 2011, 83, 6184-6190.

13. P. Papadopoulos, X. Deng, L. Mammen, D.-M. Drotlef, G. Battagliarin, C. Li, K. Müllen, K. Landfester, A. del Campo and H.-J. r. Butt, Langmuir, 2012, 28, 8392-8398.

14. M. S. Dhindsa, N. R. Smith, J. Heikenfeld, P. D. Rack, J. D. Fowlkes, M. J. Doktycz, A. V. Melechko and M. L. Simpson, Langmuir, 2006, 22, 9030-9034.

15. C. Lee and C.-J. Kim, Phys. Rev. Lett., 2011, 106, 014502.

16. T. Verho, J. T. Korhonen, L. Sainiemi, V. Jokinen, C. Bower, K. Franze, S. Franssila, P. Andrew, O. Ikkala and R. H. Ras, Proceedings of the National Academy of Sciences, 2012, 109, 10210-10213.

17. J. B. Boreyko and C.-H. Chen, Phys. Rev. Lett., 2009, 103, 174502.

18. Q. Zhang, M. He, J. Chen, J. Wang, Y. Song and L. Jiang, Chem. Commun., 2013, 49, 4516-4518.

19. N. Vourdas, C. Ranos and V. Stathopoulos, RSC Advances, 2015, 5, 33666-33673.

20. T. N. Krupenkin, J. A. Taylor, E. N. Wang, P. Kolodner, M. Hodes and T. R. Salamon, Langmuir, 2007, 23, 9128-9133. 
21. J. Wang, M. Liu, R. Ma, Q. Wang and L. Jiang, ACS Appl. Mater. Interfaces., 2014, 6, 15198-15208.

22. P. Bartlett, J. Baumberg, P. R. Birkin, M. Ghanem and M. Netti, Chem. Mater., 2002, 14, 2199-2208.

23. S. Trasatti and O. Petrii, Pure Appl. Chem., 1991, 63, 711-734.

24. M. E. Orazem and B. Tribollet, Electrochemical impedance spectroscopy, John Wiley \& Sons, 2011.

25. C. Brussieux, P. Viers, H. Roustan and M. Rakib, Electrochim. Acta, 2011, 56, 7194-7201.

26. C. Ybert, C. Barentin, C. Cottin-Bizonne, P. Joseph and L. Bocquet, Phys. Fluids, 2007, 19, 123601.

TOC Graphic

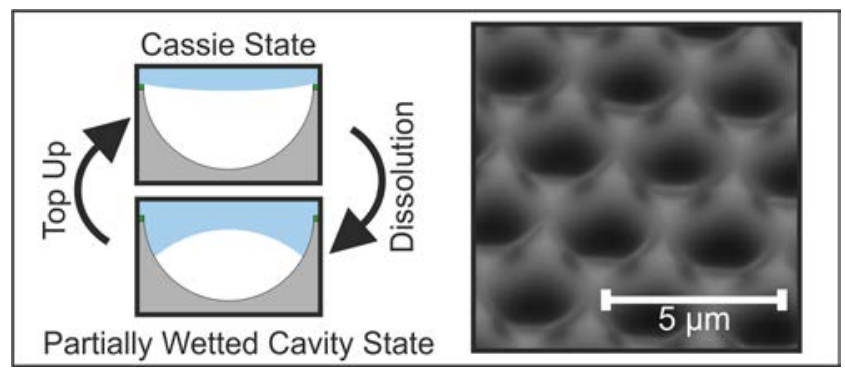

